\title{
EXISTENCE RESULTS FOR HIGHER ORDER FRACTIONAL DIFFERENTIAL EQUATIONS WITH INTEGRAL BOUNDARY CONDITIONS VIA KURATOWSKI MEASURE OF NONCOMPACTNES
}

\author{
ADEL LACHOURI ${ }^{1, a}$, ABDELOUAHEB ARDJOUNI $^{2, b}$, \\ NESRINE GOURI ${ }^{3, c}$ AND KAMEL ALI KHELIL ${ }^{4, d}$ \\ ${ }^{1}$ Laboratory of Applied Mathematics, Annaba University, Annaba, Algeria. \\ ${ }^{a}$ E-mail: lachouri.adel@yahoo.fr \\ ${ }^{2}$ Department of Mathematics and Informatics, University of Souk Ahras, P.O. Box 1553, Souk Ahras, \\ 41000, Algeria. \\ ${ }^{b}$ E-mail: abd_ardjouni@yahoo.fr \\ ${ }^{3}$ Laboratory of Mathematical Modeling and Numerical Simulation, Annaba University, Annaba, Alge- \\ ria. \\ ${ }^{b}$ E-mail: gou.nesrine@gmail.com \\ ${ }^{4}$ Laboratory of Analysis and Control of Differential Equations,University of 8 May 1945 Guelma, Algeria. \\ ${ }^{b}$ E-mail: k.alikhelil@yahoo.fr
}

\|\|$\|$

Abstract

In this work, we study the existence results for higher order fractional differential equations involving the Caputo-Hadamard fractional derivative subject to integral boundary conditions (IBCs for short). Our results are obtained by using the technique of measures of noncompactness combined with fixed point theorem of Mönch. An example demonstrating the effectiveness of the theoretical findings is presented.

\section{Introduction}

In latest years, fractional differential equations (FDEs for short) theory has received very broad regard in the fields of pure and applied mathematics, see [20, 23]. FDE's emerge naturally in diverse scopes of science, with many applications, e.g. [4, 13, 18, 24, 30].

Received June 11, 2021.

AMS Subject Classification: 34A08, 34B15, 47H08.

Key words and phrases: Fractional differential equations, Caputo-Hadamard fractional derivative, measure of noncompactness, Mönch fixed point theorem, existence, Banach space. 
Measure of noncompactness (MNC for short) combined with one of fixed point theorems, as Darbo [12] Sadovski [27], Mönch [25] is an important and efficacy tool in study of differential or integral equations. $\mathrm{Ku}$ ratowski 21] introduced the concept of MNC, which played an important role in fixed point theory, Gohberg [16] gave an other measure called Hausdorff measure later Darbo [12] used Kuratowski's MNC to generalize the Schauder's theorem of fixed point. After, that many authors studied and solved some problems by using MNC in study of different kind problems, as differential equations, integral equations and integro-differential equations, see [1, 6, 7, 8, 9, 10, 11, 17, 28].

Recently in [3], Arioua et al, studied the existence of solutions of the following problem of FDEs

$$
\left\{\begin{array}{l}
{ }_{H}^{C} \mathfrak{D}_{1}^{r_{1}} \varkappa(\tau)=q(\tau, \varkappa(\tau)), \tau \in(1, e), 2<r_{1} \leq 3, \\
\varkappa(1)=\varkappa^{\prime}(1)=0,{ }_{H}^{C} \mathfrak{D}_{1}^{r_{1}-1} \varkappa(e)={ }_{H}^{C} \mathfrak{D}_{1}^{r_{1}-2} \varkappa(e)=0,
\end{array}\right.
$$

where ${ }_{H}^{C} \mathfrak{D}_{1}^{r_{1}}$ is the fractional derivative (FD for short) in Caputo-Hadamard sense of order $r_{1}$ and $q:[1, e] \times \mathbb{R} \rightarrow \mathbb{R}$ is a given continuous function.

In [15], Duraisamy et al, used some fixed point theorems to debate the existence of solutions of higher order FDEs given by

$$
\left\{\begin{array}{l}
{ }^{C} D^{r_{1}} \varkappa(\tau)=q(\tau, \varkappa(\tau)), \tau \in[0,1], r_{1} \in(\mathfrak{n}-1, \mathfrak{n}], \mathfrak{n} \geq 2, \mathfrak{n} \in \mathbb{N}, \\
\varkappa(0)=\varkappa^{\prime}(0)=\varkappa^{\prime \prime}(0)=\cdots=\varkappa^{(\mathfrak{n}-2)}(0)=0, \\
\varkappa(1)=\sum_{i=1}^{\mathfrak{n}} \gamma_{i}\left[I^{\beta_{i}} \varkappa\left(\eta_{i}\right)-I^{\beta_{i}} \varkappa\left(\zeta_{i}\right)\right], \beta_{i}>0,
\end{array}\right.
$$

where ${ }^{C} D^{r_{1}}$ and $I^{\beta_{i}}$ are the Caputo FD and Riemann-Liouville fractional integral (FI for short)of order $r_{1}, \beta_{i}$, respectively, $0<\zeta_{1}<\eta_{1}<\ldots<\zeta_{\mathfrak{n}}<$ $\eta_{\mathfrak{n}}<1$ and $q:[0,1] \times \mathbb{R} \rightarrow \mathbb{R}$ is a given continuous function.

In [11] Boutiara et al, used the technique of MNC to study existence of solution of the following FDEs with three-point boundary conditions

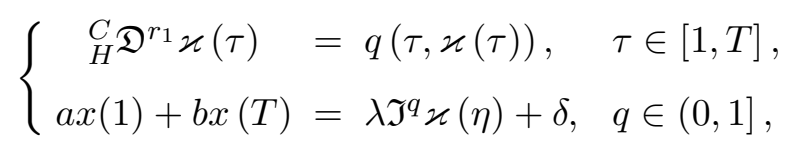

where $\mathfrak{I}^{q}$ is the Hadamard FD of order $q, 0<r_{1}, q \leq 1, q:[1, T] \times \mathcal{X} \rightarrow \mathcal{X}$ is a given continuous function, $\mathcal{X}$ is a Banach space, $a, b, \lambda \in \mathbb{R}$ and $\eta \in(1, T)$. 
Inspired and motivated by the aforementioned works, we prove the existence of mild solutions for higher order FDEs with integral boundary conditions

$$
\left\{\begin{array}{l}
{ }_{H}^{C} \mathfrak{D}_{1}^{r_{1}} \varkappa(\tau)=q(\tau, \varkappa(\tau)), \tau \in\left(1, \tau_{1}\right), r_{1} \in(\mathfrak{n}-1, \mathfrak{n}], \mathfrak{n} \geq 2, \\
\varkappa(1)=\varkappa^{\prime}(1)=\varkappa^{\prime \prime}(1)=\cdots=\varkappa^{(\mathfrak{n}-2)}(1)=0 \\
\varkappa\left(\tau_{1}\right)=\lambda \int_{1}^{\tau_{1}} \varkappa(\zeta) \frac{d \zeta}{\zeta}, \lambda \in \mathbb{R}
\end{array}\right.
$$

where $q:[a, b] \times \mathcal{X} \rightarrow \mathcal{X}$ is given continuous function satisfying some assumptions that will be specified later, and $\mathcal{X}$ be a Banach space with the norm $\|\cdot\|$.

This paper is structured as follows. In Sect. 2, we give some fundamentals ideas of fractional calculus (FC for short) and Kuratowski MNC techniques. In Sect. 3, we demonstrate the existence outcomes for (1.1) by using the fixed point theorem of Mönch. At the end, an example is given in Sect. 4.

\section{Preliminaries}

In this part, we give some fundamentals ideas of FC, Kuratowski MNC techniques and fixed point theorem that prerequisite in our analysis.

Let $J_{1}=\left[1, \tau_{1}\right]$. By $\mathcal{C}=C\left(J_{1}, \mathcal{X}\right)$ we denote the Banach space of all continuous functions $\varkappa: J_{1} \rightarrow \mathcal{X}$ with norm

$$
\|\varkappa\|_{\infty}=\sup \left\{\|\varkappa(\tau)\|: \tau \in J_{1}\right\}
$$

Let $L^{1}\left(J_{1}, \mathcal{X}\right)$ be the Banach space of measurable functions $\varkappa: J_{1} \rightarrow \mathcal{X}$ that are Lebesgue integrable with norm

$$
\|\varkappa\|_{L^{1}}=\int_{J_{1}}\|\varkappa(\tau)\| d \tau .
$$

And $A C\left(J_{1}, \mathcal{X}\right)$ be the space of absolutely continuous valued functions on $J_{1}$, and set

$$
\begin{aligned}
A C^{n}\left(J_{1}\right) & =\left\{\varkappa: J_{1} \rightarrow \mathcal{X}: \varkappa, \varkappa^{\prime}, \varkappa^{\prime \prime}, \ldots, \varkappa^{n-1} \in C\left(J_{1}, \mathcal{X}\right),\right. \\
& \text { and } \left.\varkappa^{n-1} \in A C\left(J_{1}, \mathcal{X}\right)\right\} .
\end{aligned}
$$


Furthermore, for a given set $\mathcal{V}$ of function $v: J_{1} \rightarrow \mathcal{X}$ let us denote by

$$
\mathcal{V}(\tau)=\{v(\tau): v \in \mathcal{V}\}, \tau \in J_{1}
$$

and

$$
\mathcal{V}\left(J_{1}\right)=\left\{v(\tau): v \in \mathcal{V}, \tau \in J_{1}\right\}
$$

Definition $1([20])$. The Hadamard FI of order $r_{1}>0$ for a function $\varkappa \in$ $L^{1}\left(J_{1}\right)$ is described by

$$
H \mathfrak{I}_{1}^{r_{1}} \varkappa(\tau)=\frac{1}{\Gamma\left(r_{1}\right)} \int_{1}^{\tau}\left(\log \frac{\tau}{\zeta}\right)^{r_{1}-1} \varkappa(\zeta) \frac{d \zeta}{\zeta}, r_{1}>0
$$

Set $\delta=\left(\tau \frac{d}{d \tau}\right), n=\left[r_{1}\right]+1$, where $r_{1}$ denotes the integer part of $r_{1}$. Define the space

$$
A C_{\delta}^{n}\left(J_{1}\right)=\left\{\varkappa: J_{1} \rightarrow \mathbb{R}: \delta^{n-1} \varkappa(\tau) \in A C\left(J_{1}\right)\right\}
$$

Definition 2 ([20]). The Hadamard FD of order $r_{1}>0$ for a function $\varkappa \in A C_{\delta}^{n}\left(J_{1}\right)$ is described by

$$
\begin{aligned}
{ }^{H} \mathfrak{D}_{1}^{r_{1}} \varkappa(\tau) & =\delta^{n}\left({ }^{H} \mathfrak{I}^{n-r_{1}} \varkappa\right)(\tau) \\
& =\frac{1}{\Gamma\left(n-r_{1}\right)}\left(\tau \frac{d}{d \tau}\right)^{n} \int_{1}^{\tau}\left(\log \frac{\tau}{\zeta}\right)^{n-r_{1}-1} \varkappa(\zeta) \frac{d \zeta}{\zeta}
\end{aligned}
$$

Definition 3 ([19]). The Caputo-Hadamard FD of order $r_{1}>0$ for a function $\varkappa \in A C_{\delta}^{n}\left(J_{1}\right)$ is described by

$$
\begin{aligned}
{ }_{H}^{C} \mathfrak{D}_{1}^{r_{1}} \varkappa(\tau) & =\left({ }^{H} \mathfrak{I}_{1}^{n-r_{1}} \delta^{n} \varkappa\right)(\tau) \\
& =\frac{1}{\Gamma\left(n-r_{1}\right)} \int_{1}^{\tau}\left(\log \frac{\tau}{\zeta}\right)^{n-r_{1}-1} \delta^{n} \varkappa(\zeta) \frac{d \zeta}{\zeta} .
\end{aligned}
$$

Lemma 1 ([19]). Let $r_{1}>0$ and $\mathfrak{n}=\left[r_{1}\right]+1$. If $\varkappa \in A C_{\delta}^{n}\left(J_{1}\right)$, then the Caputo-Hadamard FDE

$$
{ }_{H}^{C} \mathfrak{D}_{1}^{r_{1}} \varkappa(\tau)=0
$$

has a solution

$$
\varkappa(\tau)=\sum_{k=0}^{\mathfrak{n}-1} c_{k}(\log \tau)^{k}
$$


and the following formula holds

$$
H \mathfrak{I}_{1}^{r_{1}}\left({ }_{H}^{C} \mathfrak{D}_{1}^{r_{1}} \varkappa(\tau)\right)=\varkappa(\tau)+\sum_{k=0}^{\mathfrak{n}-1} c_{k}(\log \tau)^{k},
$$

where $c_{k} \in \mathbb{R}, k=1,2, \ldots, \mathfrak{n}-1$.

To study the nonlinear problem (1.1), we need the following lemma.

Lemma 2. Let $\Delta=\mathfrak{n}\left(\log \tau_{1}\right)^{\mathfrak{n}-1}-\lambda\left(\log \tau_{1}\right)^{\mathfrak{n}} \neq 0$. For any $\omega \in \mathcal{C}$, then the solution of boundary value problem

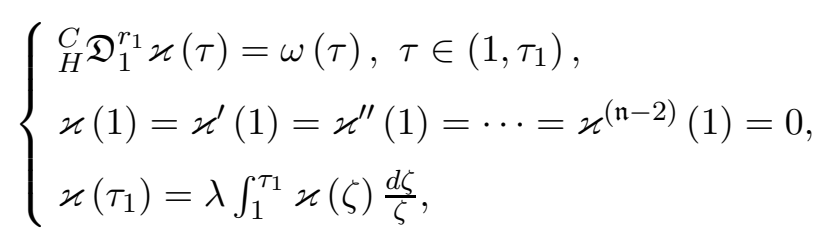

is obtained as

$$
\begin{aligned}
\varkappa(\tau)= & \frac{1}{\Gamma\left(r_{1}\right)} \int_{1}^{\tau}\left(\log \frac{\tau}{\zeta}\right)^{r_{1}-1} \omega(\zeta) \frac{d \zeta}{\zeta} \\
& +\frac{\mathfrak{n}(\log \tau)^{\mathfrak{n}-1}}{\Delta}\left(\frac{1}{\Gamma\left(r_{1}\right)} \int_{1}^{\tau_{1}}\left(\log \frac{\tau_{1}}{\zeta}\right)^{r_{1}-1} \omega(\zeta) \frac{d \zeta}{\zeta}\right. \\
& \left.-\lambda \int_{1}^{\tau_{1}}\left(\frac{1}{\Gamma\left(r_{1}\right)} \int_{1}^{\zeta}\left(\log \frac{\zeta}{\sigma}\right)^{r_{1}-1} \omega(\sigma) \frac{d \sigma}{\sigma}\right) \frac{d \zeta}{\zeta}\right)
\end{aligned}
$$

Proof. Using ${ }^{H} \mathfrak{I}_{1}^{r_{1}}$ to (2.1), and by Lemma 1, we have

$$
\varkappa(\tau)=I^{r_{1}} \omega(\tau)-c_{0}-c_{1} \log \tau-c_{2}(\log \tau)^{2}-\cdots-c_{\mathfrak{n}-1}(\log \tau)^{\mathfrak{n}-1},
$$

then

$$
\begin{aligned}
\varkappa^{\prime}(\tau)= & \frac{1}{\Gamma\left(r_{1}-1\right) \tau} \int_{1}^{\tau}\left(\log \frac{\tau}{\zeta}\right)^{r_{1}-2} \omega(\zeta) \frac{d \zeta}{\zeta}-\frac{c_{1}}{\tau}-c_{2} \frac{2 \log \tau}{\tau} \\
& -\cdots-c_{\mathfrak{n}-1} \frac{(\mathfrak{n}-1)(\log \tau)^{\mathfrak{n}-2}}{\tau}, \\
\varkappa^{\prime \prime}(\tau)= & \frac{-1}{\Gamma\left(r_{1}-1\right) \tau^{2}} \int_{1}^{\tau}\left(\log \frac{\tau}{\zeta}\right)^{r_{1}-2} \omega(\zeta) \frac{d \zeta}{\zeta}
\end{aligned}
$$




$$
\begin{aligned}
& +\frac{1}{\Gamma\left(r_{1}-2\right) \tau} \int_{1}^{\tau}\left(\log \frac{\tau}{\zeta}\right)^{r_{1}-3} \omega(\zeta) \frac{d \zeta}{\zeta} \\
& -c_{1}\left(\frac{-1}{\tau^{2}}\right)-2 c_{2}\left(\frac{1}{\tau^{2}}-\frac{\log \tau}{\tau^{2}}\right) \\
& -\cdots-(\mathfrak{n}-1) c_{\mathfrak{n}-1}\left(\frac{(\log \tau)^{\mathfrak{n}-2}}{\tau^{2}}-\frac{(\mathfrak{n}-2)(\log \tau)^{\mathfrak{n}-3}}{\tau^{2}}\right), \ldots
\end{aligned}
$$

Applying the boundary conditions, we have

$$
c_{0}=c_{1}=c_{2}=\cdots=c_{\mathfrak{n}-2}=0
$$

By substituting (2.4) in (2.3), we get

$$
\varkappa(\tau)=H \mathfrak{I}_{1}^{r_{1}} \omega(\tau)-c_{\mathfrak{n}-1}(\log \tau)^{\mathfrak{n}-1} .
$$

From the integral condition of (2.1), we have

$$
\begin{aligned}
& \frac{1}{\Gamma\left(r_{1}\right)} \int_{1}^{\tau_{1}}\left(\log \frac{\tau_{1}}{\zeta}\right)^{r_{1}-1} \omega(\zeta) \frac{d \zeta}{\zeta}-c_{\mathfrak{n}-1}\left(\log \tau_{1}\right)^{\mathfrak{n}-1} \\
& =\lambda \int_{1}^{\tau_{1}}\left(\frac{1}{\Gamma\left(r_{1}\right)} \int_{1}^{\zeta}\left(\log \frac{\zeta}{\sigma}\right)^{r_{1}-1} \omega(\sigma) \frac{d \sigma}{\sigma}\right) \frac{d \zeta}{\zeta}-\frac{\lambda c_{\mathfrak{n}-1}\left(\log \tau_{1}\right)^{\mathfrak{n}}}{\mathfrak{n}}
\end{aligned}
$$

so

$$
\begin{aligned}
c_{\mathfrak{n}-1}= & \frac{\mathfrak{n}}{\Delta}\left(\frac{1}{\Gamma\left(r_{1}\right)} \int_{1}^{\tau_{1}}\left(\log \frac{\tau_{1}}{\zeta}\right)^{r_{1}-1} \omega(\zeta) \frac{d \zeta}{\zeta}\right. \\
& \left.-\lambda \int_{1}^{\tau_{1}}\left(\frac{1}{\Gamma\left(r_{1}\right)} \int_{1}^{\zeta}\left(\log \frac{\zeta}{\sigma}\right)^{r_{1}-1} \omega(\sigma) \frac{d \sigma}{\sigma}\right) \frac{d \zeta}{\zeta}\right) .
\end{aligned}
$$

By substituting (2.6) in (2.5), we obtain (2.2).

Definition $4([2,5])$. Let $\mathcal{X}$ be a Banach space and $\Omega_{\mathcal{X}}$ the bounded subsets of $\mathcal{X}$. The Kuratowski MNC is the map $\mathfrak{m}: \Omega_{\mathcal{X}} \rightarrow[0, \infty)$ defined by

$$
\mathfrak{m}(\mathfrak{B})=\inf \left\{\epsilon>0: B \subseteq \cup_{i=1}^{n} \mathfrak{B}_{i} \text { and } \operatorname{diam}\left(\mathfrak{B}_{i}\right) \leq \epsilon\right\} \text {, here } \mathfrak{B} \in \Omega_{\mathcal{X}}
$$

This MNC satisfies some properties:

(a) $\mathfrak{m}(\mathfrak{B})=0 \Leftrightarrow \overline{\mathfrak{B}}$ is compact ( $\mathfrak{B}$ is relatively compact),

(b) $\mathfrak{m}(\mathfrak{B})=\mathfrak{m}(\overline{\mathfrak{B}})$,

(c) $\mathcal{A} \subset \mathfrak{B} \Rightarrow \mathfrak{m}(\mathcal{A}) \leq \mathfrak{m}(\mathfrak{B})$, 
(d) $\mathfrak{m}(\mathcal{A}+\mathfrak{B}) \leq \mathfrak{m}(\mathcal{A})+\mathfrak{m}(\mathfrak{B})$,

(e) $\mathfrak{m}(c \mathfrak{B})=|c| \mathfrak{m}(\mathfrak{B}), c \in \mathbb{R}$,

(f) $\mathfrak{m}(\operatorname{conv} \mathfrak{B})=\mathfrak{m}(\mathfrak{B})$.

Here $\overline{\mathfrak{B}}$ and $\operatorname{conv} \mathfrak{B}$ denote the closure and the convex hull of the bounded set $\mathfrak{B}$, respectively. For more details of $\mathfrak{m}$ and its properties, we refer to [2, 5].

Definition $5([5])$. A map $q: J_{1} \times \mathcal{X} \rightarrow \mathcal{X}$ is called Carathéodory whenever the map $\tau \rightarrow q(\tau, \varkappa)$ is measurable $\forall \varkappa \in \mathcal{X}$, and the map $\varkappa \rightarrow q(\tau, \varkappa)$ is continuous for almost all $\tau \in J_{1}$.

We need the following results, which play an important role in the achievement of the desired results in this research.

Theorem 1 ([25]). Let $\mathfrak{D}$ be a bounded, closed and convex subset of the Banach space such that $0 \in \mathfrak{D}$, and let $\Phi: \mathfrak{D} \rightarrow \mathfrak{D}$ be a continuous mapping. If the implication

$$
\mathcal{V}=\overline{\operatorname{conv}} \Phi(\mathcal{V}) \text { or } \mathcal{V}=\Phi(\mathcal{V}) \cup\{0\} \Rightarrow \mathfrak{m}(\mathcal{V})=0
$$

holds for every $\mathcal{V}$ of $\mathfrak{D}$, then $\Phi$ has a fixed point.

Lemma $3([29])$. Let $\mathcal{C}$ be a Banach space, and $\mathfrak{D} \subset \mathcal{C}$ be a bounded, closed and convex subset. Let $G$ be a continuous function on $J_{1} \times J_{1}$ and $q$ a function from $J_{1} \times \mathcal{X} \rightarrow \mathcal{X}$, which satisfies the Carathéodory conditions, and suppose there is an integrable function $\mathfrak{p}: J_{1} \rightarrow \mathbb{R}^{+}$such that, $\forall \tau \in J_{1}$ and each bounded set $\mathfrak{B} \subset \mathcal{X}$, we have

$$
\lim _{h \rightarrow 0^{+}} \mathfrak{m}\left(q\left(J_{\tau, h} \times \mathfrak{B}\right)\right) \leq \mathfrak{p}(\tau) \mathfrak{m}(\mathfrak{B}) \text {, here } J_{\tau, h}=[\tau-h, \tau] \cap J_{1}
$$

If $\mathcal{V}$ is an equicontinuous subset of $\mathfrak{D}$, then

$$
\mathfrak{m}\left(\left\{\int_{J_{1}} G(s, \tau) q(s, y(s)) d s: y \in \mathcal{V}\right\}\right) \leq \int_{J_{1}}\|G(s, \tau)\| \mathfrak{p}(s) \mathfrak{m}(\mathcal{V}(s)) d s
$$

\section{Existence Results}

In what follows, we prove existence results for (1.1) by means the Mönch fixed point theorem. 
The following hypotheses are needed to obtain our main results:

(As1) $q: J_{1} \times \mathcal{X} \rightarrow \mathcal{X}$ is a Carathéodory function.

(As2) There exist $\mathfrak{p}_{q} \in L^{1}\left(J_{1}, \mathbb{R}^{+}\right) \cap C\left(J_{1}, \mathbb{R}^{+}\right)$such that

$$
\|q(\tau, \varkappa)\| \leq \mathfrak{p}_{q}(\tau)\|\varkappa\|, \forall(\tau, \varkappa) \in J_{1} \times \mathcal{X}
$$

(As3) For each bounded set $\mathfrak{B} \subset \mathcal{X}$ and $\forall \tau \in J_{1}$, we have

$$
\lim _{h \rightarrow 0^{+}} \mathfrak{m}\left(q\left(J_{\tau, h} \times \mathfrak{B}\right)\right) \leq \mathfrak{p}_{q}(\tau) \mathfrak{m}(\mathfrak{B}), \text { here } J_{\tau, h}=[\tau-h, \tau] \cap J_{1}
$$

Theorem 2. Suppose that the hypotheses (As1)-(As3) are true. If

$$
\frac{\left(\log \tau_{1}\right)^{r_{1}} \mathfrak{p}_{q}^{*}}{\Gamma\left(r_{1}+1\right)}+\frac{\mathfrak{n}\left(\log \tau_{1}\right)^{\mathfrak{n}-1}}{|\Delta|}\left(\frac{\left(\log \tau_{1}\right)^{r_{1}} \mathfrak{p}_{q}^{*}}{\Gamma\left(r_{1}+1\right)}+\frac{|\lambda|\left(\log \tau_{1}\right)^{r_{1}+1} \mathfrak{p}_{q}^{*}}{\Gamma\left(r_{1}+2\right)}\right)<1
$$

Then, (1.1) has a mild solution on $J_{1}$.

Proof. Initially, to switch (1.1) into a fixed point problem, we consider the operator $\Phi: \mathcal{C} \rightarrow \mathcal{C}$ as

$$
\begin{aligned}
(\Phi \varkappa)(\tau)= & \frac{1}{\Gamma\left(r_{1}\right)} \int_{1}^{\tau}\left(\log \frac{\tau}{\zeta}\right)^{r_{1}-1} q(\zeta, \varkappa(\zeta)) \frac{d \zeta}{\zeta} \\
& +\frac{\mathfrak{n}(\log \tau)^{\mathfrak{n}-1}}{\Delta}\left(\frac{1}{\Gamma\left(r_{1}\right)} \int_{1}^{\tau_{1}}\left(\log \frac{\tau_{1}}{\zeta}\right)^{r_{1}-1} q(\zeta, \varkappa(\zeta)) \frac{d \zeta}{\zeta}\right. \\
& \left.-\lambda \int_{1}^{\tau_{1}}\left(\frac{1}{\Gamma\left(r_{1}\right)} \int_{1}^{\zeta}\left(\log \frac{\zeta}{\sigma}\right)^{r_{1}-1} q(\sigma, \varkappa(\sigma)) \frac{d \sigma}{\sigma}\right) \frac{d \zeta}{\zeta}\right)
\end{aligned}
$$

Clearly, the mild solution of (1.1) is a fixed point of the operator $\Phi$. Consider the nonempty bounded closed convex subset

$$
\Omega=\left\{\varkappa \in \mathcal{C}:\|\varkappa\| \leq M_{0}\right\}
$$

where $M_{0}$ is chosen such that

$$
M_{0} \geq \frac{\left(\log \tau_{1}\right)^{r_{1}} \mathfrak{p}_{q}^{*}}{\Gamma\left(r_{1}+1\right)}+\frac{\mathfrak{n}\left(\log \tau_{1}\right)^{\mathfrak{n}-1}}{|\Delta|}\left(\frac{\left(\log \tau_{1}\right)^{r_{1}} \mathfrak{p}_{q}^{*}}{\Gamma\left(r_{1}+1\right)}+\frac{|\lambda|\left(\log \tau_{1}\right)^{r_{1}+1} \mathfrak{p}_{q}^{*}}{\Gamma\left(r_{1}+2\right)}\right)
$$


with $\mathfrak{p}_{q}^{*}=\sup \left\{\mathfrak{p}_{q}(\tau): \tau \in J_{1}\right\}$. We will demonstrate that $\Phi$ satisfies the hypotheses of Theorem 1, The proof will be presented as follows.

Step 1.We demonstrate that $\Phi(\Omega) \subset \Omega$.

For $\varkappa \in \Omega$, we have

$$
\begin{aligned}
&\|(\Phi \varkappa)(\tau)\| \\
& \leq \leq \frac{1}{\Gamma\left(r_{1}\right)} \int_{1}^{\tau}\left(\log \frac{\tau}{\zeta}\right)^{r_{1}-1}\|q(\zeta, \varkappa(\zeta))\| \frac{d \zeta}{\zeta} \\
& \quad+\frac{\mathfrak{n}(\log \tau)^{\mathfrak{n}-1}}{|\Delta|}\left(\frac{1}{\Gamma\left(r_{1}\right)} \int_{1}^{\tau_{1}}\left(\log \frac{\tau_{1}}{\zeta}\right)^{r_{1}-1}\|q(\zeta, \varkappa(\zeta))\| \frac{d \zeta}{\zeta}\right. \\
&\left.\quad+|\lambda| \int_{1}^{\tau_{1}}\left(\frac{1}{\Gamma\left(r_{1}\right)} \int_{1}^{\zeta}\left(\log \frac{\zeta}{\sigma}\right)^{r_{1}-1}\|q(\zeta, \varkappa(\zeta))\| \frac{d \sigma}{\sigma}\right) \frac{d \zeta}{\zeta}\right) \\
& \leq \frac{\left(\log \tau_{1}\right)^{r_{1}} \mathfrak{p}_{q}^{*}}{\Gamma\left(r_{1}+1\right)}+\frac{\mathfrak{n}\left(\log \tau_{1}\right)^{\mathfrak{n}-1}}{|\Delta|}\left(\frac{\left(\log \tau_{1}\right)^{r_{1}} \mathfrak{p}_{q}^{*}}{\Gamma\left(r_{1}+1\right)}+\frac{|\lambda|\left(\log \tau_{1}\right)^{r_{1}+1} \mathfrak{p}_{q}^{*}}{\Gamma\left(r_{1}+2\right)}\right)
\end{aligned}
$$

and consequently

$$
\|\Phi \varkappa\|_{\infty} \leq M_{0}
$$

Hence, $\Phi(\Omega) \subset \Omega$ and the set $\Phi(\Omega)$ is uniformly bounded.

Step 2. $\Phi$ sends bounded sets of $\mathcal{C}$ into equicontinuous sets.

For $\tau_{1}, \tau_{2} \in J_{1}, \tau_{1}<\tau_{2}$ and for $\varkappa \in \Omega$, we have

$$
\begin{aligned}
\left\|(\Phi \varkappa)\left(\tau_{2}\right)-(\Phi \varkappa)\left(\tau_{1}\right)\right\| \\
\leq \frac{\mathfrak{p}_{q}^{*}}{\Gamma\left(r_{1}\right)} \int_{1}^{\tau_{1}}\left[\left(\log \frac{\tau_{2}}{\zeta}\right)^{r_{1}-1}-\left(\log \frac{\tau_{1}}{\zeta}\right)^{r_{1}-1}\right] \frac{d \zeta}{\zeta} \\
\quad+\frac{\mathfrak{p}_{q}^{*}}{\Gamma\left(r_{1}\right)} \int_{\tau_{1}}^{\tau_{2}}\left(\log \frac{\tau_{2}}{\zeta}\right)^{r_{1}-1} \frac{d \zeta}{\zeta} \\
\quad+\frac{\mathfrak{n} \mathfrak{p}_{q}^{*}\left(\left(\log \tau_{2}\right)^{\mathfrak{n}-1}-\left(\log \tau_{1}\right)^{\mathfrak{n}-1}\right)}{|\Delta|}\left(\frac{1}{\Gamma\left(r_{1}\right)} \int_{1}^{\tau_{1}}\left(\log \frac{\tau_{1}}{\zeta}\right)^{r_{1}-1} \frac{d \zeta}{\zeta}\right. \\
\left.\quad+|\lambda| \int_{1}^{\tau_{1}}\left(\frac{\mathfrak{p}_{q}^{*}}{\Gamma\left(r_{1}\right)} \int_{1}^{\zeta}\left(\log \frac{\zeta}{\sigma}\right)^{r_{1}-1} \frac{d \sigma}{\sigma}\right) \frac{d \zeta}{\zeta}\right)
\end{aligned}
$$




$$
\begin{aligned}
\leq & \frac{\mathfrak{p}_{q}^{*}}{\Gamma\left(r_{1}+1\right)}\left(\left(\log \tau_{2}\right)^{r_{1}}-\left(\log \tau_{1}\right)^{r_{1}}\right) \\
& +\frac{\mathfrak{n}\left(\left(\log \tau_{2}\right)^{\mathfrak{n}-1}-\left(\log \tau_{1}\right)^{\mathfrak{n}-1}\right)}{|\Delta|}\left(\frac{\left(\log \tau_{1}\right)^{r_{1}} \mathfrak{p}_{q}^{*}}{\Gamma\left(r_{1}+1\right)}+\frac{|\lambda|\left(\log \tau_{1}\right)^{r_{1}+1} \mathfrak{p}_{q}^{*}}{\Gamma\left(r_{1}+2\right)}\right) .
\end{aligned}
$$

As $\tau_{1} \rightarrow \tau_{2}$, we obtain

$$
\left\|(\Phi \varkappa)\left(\tau_{2}\right)-(\Phi \varkappa)\left(\tau_{1}\right)\right\| \rightarrow 0
$$

Hence $\Phi(\Omega)$ is equicontinuous.

Step 3: $\Phi$ is continuous.

Let $\left\{\varkappa_{n}\right\}$ be sequence such that $\varkappa_{n} \rightarrow \varkappa$ in $\mathcal{C}$. Then, $\forall \tau \in J_{1}$, we have

$$
\begin{aligned}
& \left\|\left(\Phi \varkappa_{n}\right)(\tau)-(\Phi \varkappa)(\tau)\right\| \\
& \leq \frac{1}{\Gamma\left(r_{1}\right)} \int_{1}^{\tau}\left(\log \frac{\tau}{\zeta}\right)^{r_{1}-1}\left\|q\left(\zeta, \varkappa_{n}(\zeta)\right)-q(\zeta, \varkappa(\zeta))\right\| \frac{d \zeta}{\zeta} \\
& \quad+\frac{\mathfrak{n}(\log \tau)^{\mathfrak{n}-1}}{|\Delta|}\left(\frac{1}{\Gamma\left(r_{1}\right)} \int_{1}^{\tau_{1}}\left(\log \frac{\tau_{1}}{\zeta}\right)^{r_{1}-1}\left\|q\left(\zeta, \varkappa_{n}(\zeta)\right)-q(\zeta, \varkappa(\zeta))\right\| \frac{d \zeta}{\zeta}\right. \\
& \left.\quad+|\lambda| \int_{1}^{\tau_{1}}\left(\frac{1}{\Gamma\left(r_{1}\right)} \int_{1}^{\zeta}\left(\log \frac{\zeta}{\sigma}\right)^{r_{1}-1}\left\|q\left(\zeta, \varkappa_{n}(\zeta)\right)-q(\zeta, \varkappa(\zeta))\right\| \frac{d \sigma}{\sigma}\right) \frac{d \zeta}{\zeta}\right) .
\end{aligned}
$$

Since $q$ is Carathéodory type, then by the Lebesgue dominated convergence theorem, we have

$$
\left\|\left(\Phi \varkappa_{n}\right)-(\Phi \varkappa)\right\|_{\infty} \rightarrow 0 \text { as } n \rightarrow \infty
$$

Now let $\mathcal{V}$ be a subset of $\Omega$ such that $\mathcal{V} \subset \overline{\operatorname{conv}}((\Phi \mathcal{V}) \cup\{0\})$. $\mathcal{V}$ is bounded and equicontinuous, and therefore the function $v \rightarrow v(\tau)=\mathfrak{m}(\mathcal{V}(\tau))$ is continuous on $J_{1}$. By assumption (As3), Lemma (3) and the properties of the measure $\mathfrak{m}$ we have for each $\tau \in J_{1}$

$$
\begin{aligned}
v(\tau) \leq & \mathfrak{m}((\Phi \mathcal{V})(\tau) \cup\{0\}) \leq \mathfrak{m}((\Phi \mathcal{V})(\tau)) \\
\leq & \frac{1}{\Gamma\left(r_{1}\right)} \int_{1}^{\tau}\left(\log \frac{\tau}{\zeta}\right)^{r_{1}-1} \mathfrak{p}_{q}(\zeta) \mathfrak{m}(\mathcal{V}(s)) \frac{d \zeta}{\zeta} \\
& +\frac{\mathfrak{n}(\log \tau)^{\mathfrak{n}-1}}{|\Delta|}\left(\frac{1}{\Gamma\left(r_{1}\right)} \int_{1}^{\tau_{1}}\left(\log \frac{\tau_{1}}{\zeta}\right)^{r_{1}-1} \mathfrak{p}_{q}(\zeta) \mathfrak{m}(\mathcal{V}(s)) \frac{d \zeta}{\zeta}\right.
\end{aligned}
$$




$$
\begin{aligned}
& \left.+|\lambda| \int_{1}^{\tau_{1}}\left(\frac{1}{\Gamma\left(r_{1}\right)} \int_{1}^{\zeta}\left(\log \frac{\zeta}{\sigma}\right)^{r_{1}-1} \mathfrak{p}_{q}(\zeta) \mathfrak{m}(\mathcal{V}(s)) \frac{d \sigma}{\sigma}\right) \frac{d \zeta}{\zeta}\right) \\
\leq & \frac{\left(\log \tau_{1}\right)^{r_{1}} \mathfrak{p}_{q}^{*}\|v\|_{\infty}}{\Gamma\left(r_{1}+1\right)}+\frac{\mathfrak{n}\left(\log \tau_{1}\right)^{\mathfrak{n}-1}}{|\Delta|}\left(\frac{\left(\log \tau_{1}\right)^{r_{1}} \mathfrak{p}_{q}^{*}\|v\|_{\infty}}{\Gamma\left(r_{1}+1\right)}\right. \\
& \left.+\frac{|\lambda|\left(\log \tau_{1}\right)^{r_{1}+1} \mathfrak{p}_{q}^{*}\|v\|_{\infty}}{\Gamma\left(r_{1}+2\right)}\right) \\
\leq & \|v\|_{\infty}\left(\frac{\left(\log \tau_{1}\right)^{r_{1}} \mathfrak{p}_{q}^{*}}{\Gamma\left(r_{1}+1\right)}+\frac{\mathfrak{n}\left(\log \tau_{1}\right)^{\mathfrak{n}-1}}{|\Delta|}\left(\frac{\left(\log \tau_{1}\right)^{r_{1}} \mathfrak{p}_{q}^{*}}{\Gamma\left(r_{1}+1\right)}\right.\right. \\
& \left.\left.+\frac{|\lambda|\left(\log \tau_{1}\right)^{r_{1}+1} \mathfrak{p}_{q}^{*}}{\Gamma\left(r_{1}+2\right)}\right)\right) .
\end{aligned}
$$

This means that

$$
\|v\|_{\infty}\left[1-\left(\frac{\left(\log \tau_{1}\right)^{r_{1}} \mathfrak{p}_{q}^{*}}{\Gamma\left(r_{1}+1\right)}+\frac{\mathfrak{n}\left(\log \tau_{1}\right)^{\mathfrak{n}-1}}{|\Delta|}\left(\frac{\left(\log \tau_{1}\right)^{r_{1}} \mathfrak{p}_{q}^{*}}{\Gamma\left(r_{1}+1\right)}+\frac{|\lambda|\left(\log \tau_{1}\right)^{r_{1}+1} \mathfrak{p}_{q}^{*}}{\Gamma\left(r_{1}+2\right)}\right)\right)\right]
$$

As (3.1), it yields to $\|v\|_{\infty}=0$, which implies that $v(\tau)=0 \forall \tau \in J_{1}$, and then $\mathcal{V}(\tau)$ is relatively compact in $\mathcal{X}$. In the light of Ascoli-Arzela theorem, $\mathcal{V}$ is relatively compact in $\Omega$. So, by the Mönch theorem, we infer that $\Phi$ has fixed point which is a mild solution of (1.1)

\section{Example}

In this portion. To validate the existence results, we consider the following FDE.

$$
\left\{\begin{array}{l}
{ }_{H}^{C} \mathfrak{D}_{1}^{\frac{5}{2}} \varkappa(\tau)=\frac{1}{3 \tau^{2}+\exp \left(\tau^{2}-1\right)} \varkappa_{n}(\tau) \\
\varkappa(1)=\varkappa^{\prime}(1)=0, \varkappa(e)=\frac{1}{2} \int_{1}^{e} \varkappa(\zeta) \frac{d \zeta}{\zeta}
\end{array}\right.
$$

Here, $r_{1}=\frac{5}{2}, \lambda=\frac{1}{2}, \tau_{1}=e, \mathfrak{n}=3$. With these data we find $\Delta=2.5 \neq 0$.

Let

$$
\mathcal{X}=l^{1}=\left\{\varkappa=\left(\varkappa_{1}, \varkappa_{2}, \ldots, \varkappa_{n}, \ldots\right): \sum_{n=1}^{\infty}\left|\varkappa_{n}\right|<\infty\right\}
$$


equipped with the norm

$$
\|\varkappa\|_{\mathcal{X}}=\sum_{n=1}^{\infty}\left|\varkappa_{n}\right|
$$

Set

$$
\begin{aligned}
\varkappa & =\left(\varkappa_{1}, \varkappa_{2}, \ldots, \varkappa_{n}, \ldots\right) \text { and } q=\left(q_{1}, q_{2}, \ldots, q_{n}, \ldots,\right), \\
q_{n}\left(\tau, \varkappa_{n}\right) & =\frac{1}{3 \tau^{2}+\exp \left(\tau^{2}-1\right)} \varkappa_{n}, \tau \in J_{1},
\end{aligned}
$$

For each $\varkappa_{n}$ and $\tau \in J_{1}$, we have

$$
\left|q_{n}\left(\tau, \varkappa_{n}\right)\right| \leq \frac{1}{3 \tau^{2}+\exp \left(\tau^{2}-1\right)}\left|\varkappa_{n}\right| .
$$

Thus, assumptions (As1) and (As2) are valid with $\mathfrak{p}_{q}(\tau)=\frac{1}{3 \tau^{2}+\exp \left(\tau^{2}-1\right)}$. By (4.2) and for any bounded set $\mathfrak{B} \subset l^{1}$, we have

$$
\mathfrak{m}(q(\tau, \mathfrak{B})) \leq \frac{1}{3 \tau^{2}+\exp \left(\tau^{2}-1\right)} \mathfrak{m}(\mathfrak{B}) \text { for each } \tau \in J_{1},
$$

Hence (As3) is satisfied. The condition

$$
\frac{\left(\log \tau_{1}\right)^{r_{1}} \mathfrak{p}_{q}^{*}}{\Gamma\left(r_{1}+1\right)}+\frac{\mathfrak{n}\left(\log \tau_{1}\right)^{\mathfrak{n}-1}}{|\Delta|}\left(\frac{\left(\log \tau_{1}\right)^{r_{1}} \mathfrak{p}_{q}^{*}}{\Gamma\left(r_{1}+1\right)}+\frac{|\lambda|\left(\log \tau_{1}\right)^{r_{1}+1} \mathfrak{p}_{q}^{*}}{\Gamma\left(r_{1}+2\right)}\right) \simeq 0.18<1
$$

where $\mathfrak{p}_{q}^{*}=\sup _{\tau \in J_{1}} \mathfrak{p}_{q}(\tau)=\frac{1}{4}$. Then, in the light of Theorem 2, we infer that the problem (4.1) has at least one mild solution on $[1, e]$.

\section{References}

1. R. P. Agarwal, M. Benchohra and D. Seba, On the application of measure of noncompactness to the existence of solutions for fractional differential equations, Results Math, 55 (2009), No. 3-4, 221-230.

2. R. R. Akhmerov, M. I. Kamenskii, A. S. Potapov, A. E. Rodkina and B. N. Sadovskii, Measures of Noncompactness and Condensing Operators, Oper. Theory Adv. Appl. 55, Birkhäuser, Basel, 1992.

3. Y. Arioua and N. Benhamidouche, Boundary value problem for Caputo-Hadamard fractional differential equations, Surveys in Mathematics and its Applications, 12 (2017), 103-115. 
4. D. Baleanu, J. A. T. Machado and A. C. J. Luo, Fractional Dynamics and Control, Springer, New York, 2002.

5. J. Banaś and K. Goebel, Measures of noncompactness in Banach spaces, Lect. Notes Pure Appl. Math. 60, Marcel Dekker, New York, 1980.

6. J. Banas and K. Sadarangani, On some measures of noncompactness in the space of continuous functions, Nonlinear Analysis: Theory, Methods. Appl, 68(2008), No. 2, 377-383.

7. M. Belmekki and K. Mekhalfi. On fractional differential equations with statedependent delay via Kuratowski measure of noncompactness, Filomat, 31(2017), No. 2, 451-460.

8. M. Benchohra, J. Henderson and D. Seba, Measure of noncompactness and fractional differential equations in Banach spaces, Commun. Appl. Anal., 12 (2008), No. 4, 419427.

9. M. Benchohra, G. M. N'Guérékata and D. Seba, Measure of noncompactness and nondensely defined semilinear functional differential equations with fractional order. Cubo, 12(2010), No.3, 35-48.

10. W. Benhamida, J. R. Graef and S. Hamani, Boundary value problems for fractional differential equations with integral and anti-periodic conditions in a Banach space, Prog. Frac. Differ. Appl., 4 (2018), No. 2, 1-7.

11. A. Boutiara, K. Guerbati and M. Benbachir, Caputo-Hadamard fractional differential equation with three-point boundary conditions in Banach spaces, Aims Mathematics, 5(2019), No. 1, 259-272.

12. G. Darbo, Punti uniti dans transformazioni un condomino non compatto, Rend. Univ Padova, 24 (1955), 84-92.

13. L. Debnath, Recent applications of fractional calculus to science and engineering, Int. J. Math. Math. Sci., 54 (2003), 3413-3442.

14. C. Derbazi, Nonlinear sequential Caputo and Caputo-Hadamard fractional differential equations with Dirichlet boundary conditions, Kragujevac Journal of Mathematics, 46(2022), No. 6, 841-855.

15. P. Duraisamy and T. Nandha Gopal, Some existence results for higher order fractional differential equations with multi-strip Riemann-Liouville type fractional integral boundary conditions, Journal of Nonlinear Analysis and Application, 2019 (2019), No. 1, 1-11.

16. I. Gohberg, Measures of Noncompactness in Metric Fixed Point Theory, Oper. Theo. Adv. Appl, 99 (1997).

17. A. Hamrouni, S. Beloul and A Aissaoui, Existence of solutions for boundary value problems of fractional integro-differential equations with integral boundary conditions on Banach spaces, Nonlinear Studies, 26 (2019), No. 3, 693-701.

18. R. Hilfer, Applications of Fractional Calculus in Physics, World Scientific, Singapore, 2000 . 
19. F. Jarad, T. Abdeljawad and D. Baleanu, Caputo-type modification of the Hadamard fractional derivatives, Advances in Difference Equations, 2012(2012), No. 142, 1-8.

20. A. A. Kilbas, H. M. Srivastava and J. J. Trujillo, Theory and Applications of Fractional Differential Equations, Elsevier Science B. V., Amsterdam, 2006.

21. K. Kuratowski, Sur les espaces complets, Fundamenta Mathematicae, 15 (1930), 301309.

22. A. Lachouri, A. Ardjouni, A. Djoudi . Existence results for integral boundary value problems of fractional differential equations with two nonlinear terms in banach spaces, Bull. Int. Math. Virtual Inst, 11 (2021), No. 1, 159-168.

23. V. Lakshmikantham, S. Leela and J. V. Devi, Theory of Fractional Dynamic Systems, Cambridge Scientific Publishers, Cambridge, UK, 2009.

24. R. Magin, Fractional calculus in bioengineering, Critical Rev. Biomed. Eng., 32 (2004), $1-104$.

25. H. Mönch, Boundary value problems for nonlinear ordinary differential equations of second order in Banach spaces, Nonlinear Anal., 4 (1980), No. 5, 985-999.

26. M. Mursaleen, B. Bilalov and S. M. H. Rizvi, Applications of measures of noncompactness to infinite system of fractional differential equations, Filomat, 31 (2017), No. 11, 3421-3432.

27. B. N. Sadovskii, On a fixed point principle, Funct. Anal. Appl., 1 (1967), 74-76.

28. D. Seba, H. Rebai and J, Henderson, Existence result for nonlinear fractional differential equations with nonlocal fractional integro differential boundary conditions in Banach spaces, Georgian Math. J., 28 (2019), No. 1, 1-7.

29. S. A. Szufla, On the application of measure of noncompactness to existence theorems, Rend. Semin. Mat. Univ. Padova., 75 (1986), 1-14.

30. V. E. Tarasov, Fractional Dynamics: Application of Fractional Calculus to Dynamics of Particles, Fields and Media, Springer, New York, 2011. 